\title{
Taille des particules alimentaires dans le canal et entre les lames du feuillet, et dans les réservoirs amont et aval chez le bouvillon
}

\author{
C Dardillat, R Baumont
}

INRA Theix, station de recherches sur la nutrition des herbivores, 63122 St-Genès-Champanelle, France

\begin{abstract}
Summary - Particle size of the digesta between the omasal leaves, in the omasal canal, in the reticulum and in the abomasum in steers. The particle size distribution of digesta taken at slaughter of grazing steers showed a higher proportion of small particles in the interlaminal space of the omasum and in the duodenum than in the omasal canal and abomasum with the smallest proportion in the reticulum. A larger retention time in the abomasum for large particles than for smalt ones and liquids was assumed, as well as in the omasal canal; but a backflow of large particles to the reticulum can also occur.
\end{abstract}

On ne trouve pratiquement pas de particules alimentaires de taille supérieure à $2 \mathrm{~mm}$ au-delà du réticulorumen chez le Bovin (Grenet, 1970). Deux mécanismes entraînent cette rétention sélective (Reid, 1984; Dardillat et Baumont, 1988) : la flottaison des grandes particules sous l'effet des gaz de fermentation qui les éloigne de l'orifice réticulo-omasal (ORO); une filtration du contenu par les plus longues particules immobilisées contre l'ORO pendant les débits. De plus, des reflux à travers l'ORO ont été observés (Mac Bride et al, 1984; Dardillat, 1987) et il n'est pas impossible que des grandes particules ayant franchi l'ORO et s'étant accumulées contre les papilles du canal ou le bord des lames omasales soient périodiquement refoulées vers le réseau. Pour tester cette hypothèse, nous avons comparé la taille des particules dans le canal et entre les lames du feuillet, dans le réseau, la caillette et le duodénum.

Matériel et méthodes - Les prélèvements de contenus stomacaux ont été réalisés à l'abattage sur 9 bouvillons de $500 \pm$ $15 \mathrm{~kg}$ venant du pâturage, après $18 \mathrm{~h}$ de jeûne. Les tamisages ont été effectués par voie humide (Grenet, 1970) avec les tamis de $4,2,1,0,4,0,16$ et $0,05 \mathrm{~mm}$. Les résultats sont exprimés en pourcentage de la masse totale séchée des particules retenues sur les tamis afin de comparer plus aisément le chyme duodénal, dont le très faible contenu en particules est dû à leur dispersion dans les sucs digestifs, avec les autres contenus stomachaux plus riches en particules.

Résultats et discussion - Les particules retenues sur les tamis de 4 et $2 \mathrm{~mm}$ sont significativement plus abondantes dans le canal qu'entre les lames 
Tableau I. Teneur en MS du contenu et répartition des particules selon leur taille (en $\%$ de la masse totale des particules) dans les réservoirs gastriques $(n=9)$.

\begin{tabular}{|c|c|c|c|c|c|c|}
\hline \multicolumn{7}{|c|}{ Omasum } \\
\hline & & Réticulum & Canal & Lames & Abomasum & Duodénum \\
\hline \multicolumn{2}{|l|}{ MS \% } & $9,3 \pm 2,2^{a}$ & $14,2 \pm 1,8^{b}$ & $20,1 \pm 2^{c}$ & $11,6 \pm 3,1^{a b}$ & $8,3 \pm 2,5^{a}$ \\
\hline $\begin{array}{c}\text { Tamis } \\
\text { " }\end{array}$ & $\begin{array}{l}4 \quad+2 \\
1 \quad+0,4 \\
0,16+0,05\end{array}$ & $\begin{array}{l}19,9 \pm 6,7^{\mathrm{a}} \\
44,9 \pm 5,3^{\mathrm{a}} \\
35,2 \pm 6,4^{\mathrm{a}}\end{array}$ & $\begin{array}{r}9,2 \pm 3,4^{\mathrm{bd}} \\
36,1 \pm 7,4^{\mathrm{bc}} \\
54,7 \pm 6,9^{\mathrm{bd}}\end{array}$ & $\begin{array}{r}5,3 \pm 2,4^{c} \\
30,1 \pm 7,3^{\mathrm{cd}} \\
64,6 \pm 7,1^{\mathrm{c}}\end{array}$ & $\begin{array}{l}12,9 \pm 7,1^{d} \\
37 \pm 5,5^{\text {bd }} \\
50,1 \pm 8,8^{d}\end{array}$ & $\begin{aligned} 6,4 & \pm 2,4^{b c} \\
32,6 & \pm 11,2^{b c} \\
61 & \pm 12^{b c}\end{aligned}$ \\
\hline
\end{tabular}

Les nombres suivis de lettres différentes sont significativement différents $(P<0,05)$.

omasales; leur pourcentage est approximativement doublé de même qu'entre le canal et le réticulum. Bien que le contenu qui sort du réticulum soit "filtré " et donc appauvri en MS, la teneur en MS du canal omasal est beaucoup plus élevée et correspond à un contenu dont le liquide a été exprimé en partie. Entre les lames omasales, l'absorption d'eau accentue cette déshydratation (Edrise et al, 1977). Le contenu duodénal est significativement plus pauvre en particules retenues sur les tamis de 2 et $4 \mathrm{~mm}$ que le contenu abomasal. II est exclu d'envisager une réduction mécanique ou chimique appréciable de la taille des particules dans l'abomasum, compte tenu du temps de séjour réduit, d'un brassage limité et d'une résistance élevée des fibres ligno-cellulosiques aux sucs digestifs. En conséquence, on doit penser que les grandes particules séjournent plus longtemps dans l'abomasum que les fines particules. La taille des particules dans le duodénum représente l'effluent abomasal et donc également l'effluent omasal, elle est très proche de celle observée entre les lames omasales. Par contre, le contenu du canal est différent de l'effluent omasal, ce qui corrobore l'hypothèse de reflux omaso-réticulaire. Mais il est éga- lement possible que les grandes particules accumulées dans le canal omasal y séjournent de façon prolongée. Cette dernière hypothèse a été proposée par Lechner-Doll et Engelhardt (1989) chez le dromadaire pour expliquer une remarquable abondance de grandes particules dans le compartiment 3 alors que l'effluent de ce compartiment n'en contient pas.

En conclusion, la taille des particules dans le canal omasal et l'existence de reflux suggèrent un refoulement sélectif des grandes particules vers le réseau, dont le mécanisme reste à démontrer.

Dardillat C (1987) Reprod Nutr Dév 27, 231. 232

Dardillat C, Baumont R (1988) Reprod Nutr Dév 28, 137-138

Edrise BM, Smith RH, Buttle HL (1977) Proc Nutr Soc 36, $8 \mathrm{~A}$

Grenet E (1970) Ann Biol Anim Biochim Biophys 10, 643-657

Lechner-Doll M, Engelhardt WV (1989) $J$ Anim Physiol Anim Nutr 61, 120-128

McBride BW, Milligan LP, Turner BV (1984) Can J Anim Sci 64 (suppl) 84-85

Reid CSW (1984) In : Ruminant physiology, concepts and consequences (Baher SK, Gawthorne JM, Mackintosh JB, Purser $D B$, eds), Proc Symp Univ Western Australia, Sydney, 79-84 